Referencia para citar este artículo: Espinosa-Herrera, G. \& Castellanos-Obregón, J. M. (2018). Procesos de estructuración de prácticas trasgresoras asociadas al consumo de sustancias psicoactivas en universitarios. Revista Latinoamericana de Ciencias Sociales, Niñez y Juventud, 16(2), 777-795. doi:https://doi.org/10.11600/1692715x.16209

\title{
Procesos de estructuración de prácticas trasgresoras asociadas al consumo de sustancias psicoactivas en universitarios*
}

\author{
GRETEL ESPINOSA-HERRERA ** \\ Profesora Universidad de Caldas, Colombia. \\ JuAN Manuel CASTEllanos-OBREGóN ${ }^{* * * *}$ \\ Profesor Universidad de Caldas, Colombia.
}

\section{Artículo recibido en julio 17 de 2017; artículo aprobado en noviembre 17 de 2017 (eds.)}

- Resumen (descriptivo): los jóvenes contemporáneos se encuentran situados culturalmente en escenarios donde la forma de ver y vivir el mundo está influenciada por las dinámicas de consumo, entre ellas de estupefacientes. Cada vez se evidencia una progresiva normalización del consumo de sustancias psicoactivas, extendiéndose de manera abierta a todos los escenarios posibles, entre ellos la universidad; contexto donde el alcohol, los psicofármacos y la marihuana (principalmente), así como la cocaína y las drogas sintéticas (en menor medida), son accesibles en la cotidianidad de muchos de ellos y de sus contextos de socialización. En este artículo se exponen los resultados de una investigación que indagó por la manera cómo se estructuran las prácticas de consumo de sustancias psicoactivas entre universitarios, centrando la atención en las intenciones que se asocian al consumo y en la interacción como proceso estructurante de las prácticas sociales. Desarrolla un diseño cualitativo, apoyado en grupos focales y entrevistas, que rescata un modelo metodológico hermenéutico, específicamente de la doble hermenéutica propuesta por Giddens (2003). Los análisis permiten concluir que las prácticas de consumo de sustancias psicoactivas se van estructurando a partir de la interrelación que se establece entre los elementos estructurales e individuales. Esta interacción determina los cursos de acción y el tipo de experiencias que viven los estudiantes en torno a las sustancias psicoactivas. Estas prácticas establecen saberes y crean nuevas expresiones y formas de relacionarse con otros miembros del grupo.

Palabras clave: joven, universidad, sustancia alucinógena, uso (Tesauro de Ciencias Sociales de la Unesco).

\footnotetext{
* El presente artículo de investigación científica y tecnológica se deriva del trabajo sobre consumo de sustancias psicoactivas en Universitarios que se desarrolló para los estudios del Doctorado en Ciencias Sociales, Niñez y Juventud (Universidad de Manizales-Cinde). Nombre del proyecto: «Estructuración de las prácticas de consumo de sustancias psicoactivas en estudiantes universitarios». Metodología mixta. Proyecto inscrito en la Vicerrectoría de Investigaciones de la Universidad de Caldas con código: 0701015. Fecha de inicio: abril 24 de 2015 , fecha de culminación: octubre 31 de 2016. Área de conocimiento: Sociología; subárea: Otras Sociologías Específicas.

** Doctora en Ciencias Sociales. Niñez y Juventud de la Universidad de Manizales-Cinde. Profesora Auxiliar del Departamento de Antropología y Sociología de la Universidad de Caldas. Investigadora del Grupo de Investigación «Comunicación, Cultura y Sociedad» (Universidad de Caldas). Orcid: 0000-0003-1589-1304. Índice H5: 1. Correo electrónico: gretel.espinosa@ucaldas.edu.co

**** Doctor en Ciencias Sociales, Niñez y Juventud de la Universidad de Manizales-Cinde. Profesor Asociado del Departamento de Antropología y Sociología de la Universidad de Caldas. Investigador del Grupo de Investigación «Comunicación, Cultura y Sociedad» (Universidad de Caldas). Orcid: 0000-0002-1720-5278. Índice H5: 4. Correo electrónico: juan.castellanos@ucaldas.edu.co
} 


\section{Structuring process of transgressing practices related to the consumption of psychoactive substances in university students}

- Abstract (descriptive): Contemporary young people are immersed within culturally-based scenarios in which their ways of living are influenced by the dynamics of drug consumption and trade. There is evidence of progressive normalization of the consumption of psychoactive substances among young people, in public spaces and in a range of scenarios, including university campuses. In this location, several psychoactive substances are consumed by young people, including primarily alcohol, psychiatric medications and marijuana and to a lesser extent cocaine and synthetic drugs, making these spaces a part of their everyday lives and as an essential place for the context of socialization. This article covers findings from a research study designed to explore how the practices of consumption of psychoactive substances were structured in university students, specifically with their intentions related to consumption and interaction as a structuring process in social practices. A qualitative design was implemented using focus groups and interviews along with a methodological-hermeneutic approach based on the double hermeneutic proposed by Giddens (2003). The analysis identified that the practices of psychoactive substance consumption are structured based upon the interrelation between structural and individual elements that determine actions and the types of experiences that students have during psychoactive substances-related contexts. These practices establish knowledge and create new expressions and ways of interacting with other members of the group.

Key words: Young person, University, Hallucinogenic substance, Use (Unesco Social Sciences Thesaurus).

\section{Processos de estruturação de práticas de transgressoras associadas ao consumo de substâncias psicocativas na universidade}

- Resumo (descritivo): Os jovens contemporâneos estão culturalmente situados em cenários onde o modo de ver e viver o mundo é influenciado pela dinâmica do consumo, incluindo narcóticos. É evidente uma normalização progressiva do consumo de substâncias psicoativas, que se estende para todos os cenários possíveis, incluindo a universidade; na qual álcool, drogas psicotrópicas e maconha (principalmente), bem como cocaína e drogas sintéticas (em menor grau), são acessíveis no cotidiano de muitos jovens e seus contextos de socialização. Este artigo apresenta os resultados de uma investigação que buscou a forma como as práticas de consumo de substâncias psicoativas entre estudantes universitários são estruturadas, focalizando nas intenções associadas ao consumo e à interação como processo estruturante de práticas sociais. Desenvolve-se um desenho qualitativo apoiado por grupos focais e entrevistas que resgatam um modelo metodológico hermenêutico, especificamente da hermenêutica dupla proposto por Giddens (2003). As análises permitiram concluir que as práticas de consumo de substâncias psicoativas são estruturadas com base na interação que se estabelece entre os elementos estruturais e os elementos individuais. Essa interação determina os cursos de ação eo tipo de experiências que os alunos vivem com substâncias psicoativas. Essas práticas estabelecem conhecimentos e criam novas expressões e formas de se relacionar com outros membros do grupo.

Palavras-chave: jovem, universidade, substância alucinógena, uso (Thesaurus de Ciências Sociais da Unesco).

-1. Introducción. -2. El consumo de sustancias psicoactivas desde la estructuración de las prácticas sociales. Anthony Giddens. -3. Metodología. -4. Resultados: una experiencia de estructuración de las prácticas de consumo de sustancias psicoactivas entre universitarios. $\mathbf{- 5}$. Conclusiones sobre los procesos de estructuración de las prácticas asociadas al consumo de sustancias psicoactivas en universitarios. - Lista de referencias. 


\section{Introducción}

La dinámica contemporánea del comercio y uso de drogas muestra que existe un desplazamiento de los usos restringidos, anteriormente regulados y con significación cultural, hacia la aparición de nuevas prácticas sociales de uso y consumo de sustancias y métodos cada vez más diversos disponibles en el mercado (Franey, 1998; Fundación Universitaria del Área Andina, 2011; Henao, 2010). Sobre el tema Henao (2010) plantea:

la revolución tecnológica permitió aislar químicamente los principios activos de las plantas psicoactivas, lo que hizo posible su manipulación y producción industrial, así como también la creación y difusión de nuevos mecanismos de consumo relacionados con la jeringuilla hipodérmica, el cigarrillo fabricado en máquinas, nuevos mecheros y fósforos. En muchas sociedades se pasó de mascar hojas de coca a inhalar cocaína, de comer o fumar opio a inyectarse morfina, y de fumar puros o pipas a cigarrillos empaquetados; estos cambios son ejemplos de las transformaciones técnicas y económicas que describen el desplazamiento del uso tradicional por el consumo contemporáneo (p. 90).

Es evidente también una progresiva normalización ${ }^{1}$ del consumo de sustancias psicoactivas entre los jóvenes, lo que se extiende de manera abierta a todos los escenarios posibles, entre ellos la universidad. En el Informe Mundial sobre drogas 2013 (Oficina de las Naciones Unidas contra la droga y el delito [UNODC], 2013), la UNODC destaca el crecimiento del mercado en las nuevas sustancias psicoactivas comercializadas como drogas legales y drogas de diseño, asimismo cómo ha aumentado a nivel mundial el abuso de medicamentos por receta médica al tiempo que continúa el uso de drogas conocidas como la marihuana, la heroína y la cocaína. En este sentido, el II Estudio Epidemiológico Andino sobre consumo de drogas en población universitaria colombiana, señala que el 33.9\% de los universitarios declaró haber usado drogas como marihuana, cocaína, LSD y éxtasis entre otras; mientras que el 85\% reportaron el consumo de bebidas alcohólicas y el $19 \%$ el uso de tabaco (Comisión Interamericana para el Control del Abuso de Drogas/OEA, 2013). A nivel mundial, en los últimos tres años se ha observado una tendencia general a la estabilización del consumo de marihuana. Sin embargo, en algunas subregiones, especialmente de América del Norte y Europa occidental y central, ese consumo ha aumentado (UNODC, 2016).

Al comparar los hallazgos de estas investigaciones con estudios anteriores, se evidencia un incremento del $61.1 \%$ en el uso del alcohol (principalmente entre las mujeres). El uso de marihuana mostró una variación estadísticamente significativa desde $11.2 \%$ en el año 2009 a 15\% en 2012, situación más evidente entre los hombres. Los datos muestran una variación en el consumo de LSD desde $1.7 \%$ en 2009 a $4.9 \%$ en 2012 y el consumo de sustancias como el hachís pasó de $0.8 \%$ en 2009 a 2.5\% en 2012 (Comisión Interamericana para el Control del Abuso de Drogas/OEA, 2013). Esta situación es común a muchas universidades regionales donde aparece la tendencia al aumento en el consumo de sustancias psicoactivas, en particular el consumo de marihuana.

En este orden de ideas, el contexto universitario, compuesto en su mayoría por población joven, es un escenario en el que al mismo tiempo que se dan procesos académicos; así, los estudiantes tienen experiencias propias del mundo juvenil entre las que aparecen este tipo de consumos (CastellanosObregón \& Espinosa-Herrera, 2013). Es importante mencionar que en la revisión bibliográfica realizada sobre el tema, autores y estudios evidencian que el contacto con las sustancias psicoactivas ocurre cada vez a más temprana edad, ubicándose, con frecuencia, a edades anteriores a la entrada a los estudios universitarios, a una edad media de 13 años para sustancias como el alcohol y el tabaco. El inicio precoz se relaciona con el escalamiento que se produce en el tipo de sustancia que se consume al momento de vincularse a niveles superiores de educación o al mundo del trabajo. En este sentido, el porcentaje de consumidores aumenta con la edad, siendo el intervalo comprendido entre los 16 y los 18 años en el que se produce el mayor incremento (Fernández-Bustos, 2010; García-Moreno, 2002;

1 Entendamos normalización como prácticas rutinarias y recurrentes de acción que estructuran la vida cotidiana de los sujetos-agentes y que contribuyen a la producción y reproducción social (Giddens, 2003). 
Instituto de Adicciones de Madrid, 2012; López, Santín, Torrico, \& Rodríguez, 2003; Patiño, 2012).

En la revisión inicial se reconocieron vacíos de conocimiento frente al tema, identificando que los estudios realizados en contextos universitarios relacionados con el consumo de sustancias psicoactivas habitualmente se centran en describir, mediante metodologías cuantitativas, la frecuencia de estos consumos, los tipos de sustancias, la relación con factores como el rendimiento académico o la deserción estudiantil y las características de los sujetos consumidores, entre otros aspectos. Así, considerando la importancia del tema, esta investigación se preguntó por ¿cómo se estructuran las prácticas de consumo de sustancias psicoactivas en un grupo de estudiantes de una universidad regional colombiana?

Este estudio reviste valor sociológico porque pone en discusión la normalización, cada vez más evidente, de las prácticas de consumo de sustancias psicoactivas entre universitarios, al mismo tiempo que se agencian políticas prohibicionistas para las cuales el consumo se ubica como una patología o un problema de criminalidad. Este trabajo invita a replantear el manejo externo del tema, en tanto actualmente las medidas de abordaje se centran en educar respecto a lo «nocivo» de estas sustancias y sancionar el consumo, lo que no responde adecuadamente a las maneras como los estudiantes -que se inician o son consumidores habituales- lo comprenden y producen, en tanto conjunto de prácticas socialmente estructuradas.

Asimismo, es un estudio que aporta nuevos conocimientos sobre las prácticas de consumo de estupefacientes entre universitarios, considerando los discursos de los actores (estudiantes) involucrados, de forma que se detallan las motivaciones sociales que sostienen el consumo, esperando que los resultados de la investigación puedan aplicarse en otras universidades, a fin de poder validar su utilidad en un estudio comparativo.

\section{El consumo de sustancias psicoactivas desde la estructuración de las prácticas sociales. Anthony Giddens}

Algunos elementos del modelo teórico de la estructuración, propuestos por el sociólogo Anthony Giddens, permitieron la construcción de un dispositivo de reflexión conceptual sobre la problemática que se presenta. El consumo de sustancias psicoactivas se ubica en el mundo de lo cotidiano, un mundo donde la relación acción (agente social) y estructura ha sido vista por las corrientes funcionalistas como un elemento de subordinación del primero al segundo. En este sentido, Giddens (2003) nos plantea una relación diferente y expresa que en el mundo de lo cotidiano la estructura social enuncia una forma de vida que se encuentra en correspondencia con la acción de los sujetos, ya que no es una relación de subordinación, ni puede verse la estructura como algo preestablecido o inmóvil; por el contrario, la estructura social debe pensarse como movimiento, como riqueza relacional que interviene, como un marco de referencia, en la acción de los agentes.

Al situar la acción de consumir sustancias psicoactivas en el marco de la vida cotidiana, podemos entenderla como «un flujo continuo de experiencias vividas (...) que pertenecen a colectividades o comunidades sociales (...) y que se da en la copresencia de dos o varios agentes que comparten en ese momento una posición y una situación particular» (Giddens, 1987, pp. 76-77). Este flujo continuo de experiencias se evidencia en el escenario de las prácticas sociales; prácticas que, al ser recurrentes en el tiempo, se consideran elementos estructurantes del mundo social. Así, las prácticas de consumo de sustancias psicoactivas entre universitarios se vuelven comprensibles desde la interacción de las acciones individuales de los actores que participan en ellas, las cuales involucran las motivaciones y las intenciones de los agentes. Asimismo, las prácticas llevan implícita una estructura que se hace evidente a partir de la utilización de las reglas y los recursos que intervienen en la interacción; determinando las relaciones simétricas o disimétricas que se generan en los contextos de las prácticas. Lo anterior se resume en la ilustración 1. 


\section{Ilustración 1. Estructuración de las prácticas sociales}

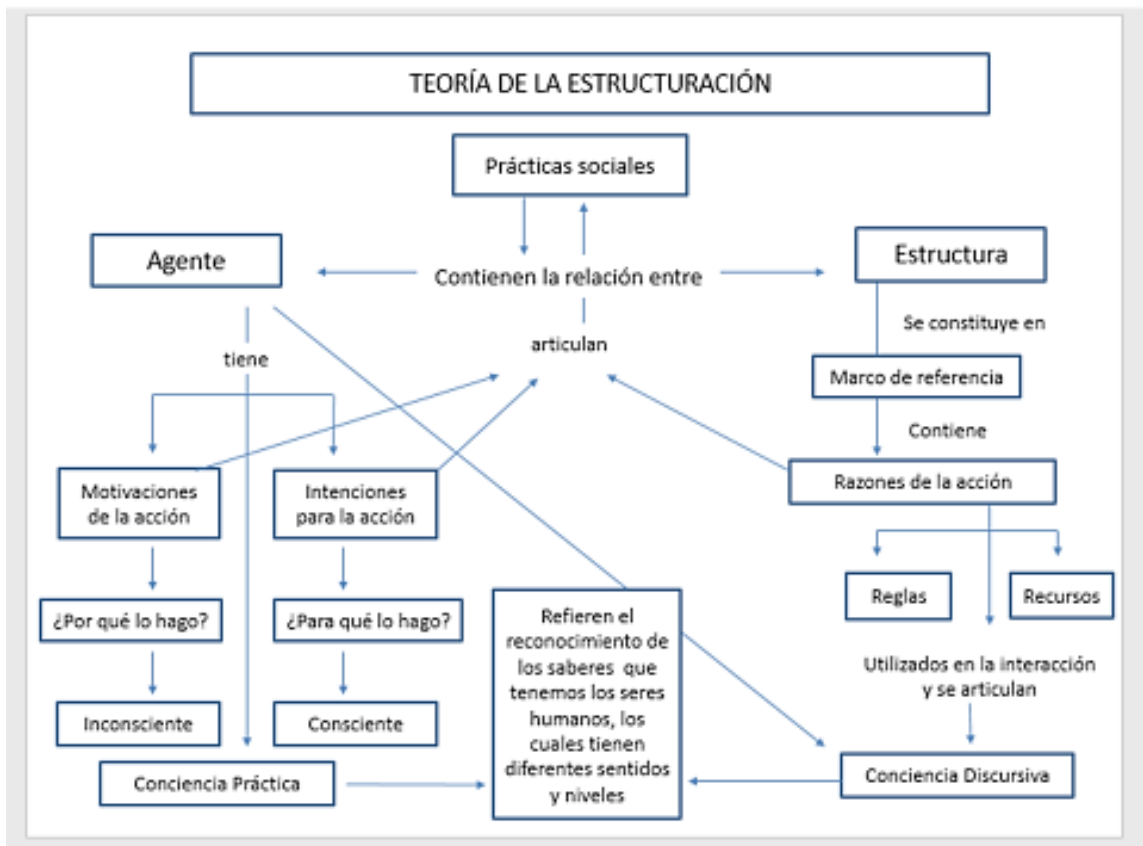

Nota. Elaborada a partir de Giddens (2003).

\section{Metodología}

Esta investigación propuso un estudio descriptivo-interpretativo de los procesos de estructuración de consumo de sustancias psicoactivas, considerando un diseño cualitativo de corte hermenéutico. Este diseño recoge y analiza datos cualitativos, a saber, los discursos de los participantes. Si bien la investigación que lo soporta tuvo un modelo general mixto, cuali-cuanti, (Creswell \& Plano, 2011), este artículo solamente presenta resultados de la fase cualitativa ${ }^{2}$, realizada en el segundo momento de la investigación, la que fue guiada por el análisis de la doble hermenéutica, orientado a entender el sentido de las configuraciones y correlaciones identificadas a la luz del dispositivo de reflexión conceptual elegido. Inicialmente se operacionalizaron los conceptos y se realizó la codificación de la información recolectada a partir de la combinación de técnicas utilizadas: la observación participativa, los grupos de enfoque y las entrevistas individuales semiestructuradas para lograr una mayor profundización en los aspectos subjetivos asociados a las prácticas de consumo entre universitarios.

La fase cualitativa se realizó durante dos años. La observación inicial se realizó siguiendo la ruta sugerida por un informante que interactúa en los espacios de la universidad y que comentó sobre los territorios vetados, las preferencias del consumidor universitario y las dinámicas dentro de estos. Con el ejercicio de observación se identificaron algunos estudiantes consumidores activos, los territorios donde los jóvenes consumen, los momentos del día, las actividades que se articulan a ese consumo, los temas de conversación entre ellos y la particularidad de los lenguajes, entre otros elementos. A partir de la observación en los espacios de consumo, nos acercamos a algunos estudiantes, a quienes invitamos a participar en el estudio y se les solicitó, a modo de muestreo de cadena o por redes «bola de nieve» (Hernández-Sampieri, Fernández-Collado, \& Baptista-Lucio, 2014, p. 388), identificar a otras personas que quisieran participar en el trabajo. Con esta estrategia se esperaba incrementar, en forma considerable, la probabilidad ampliar las características de la población estudiantil y se consideró

2 Para ampliar sobre la fase cuantitativa del estudio puede consultarse Espinosa-Herrera, Castellanos-Obregón y Osorio-García (2016). Condición juvenil y drogas en universitarios: el caso de una universidad regional. Revista Latinoamericana de Ciencias Sociales, Niñez y Juventud, 14(2), 1451-1468. 
pertinente en tanto el consumo de drogas es una práctica prohibida y no es fácil el acceso hacia la población consumidora y a sus experiencias, de manera abierta y pública. Los estudiantes invitados pertenecían a diferentes programas académicos de la universidad y como criterio de selección se exigió que estuviesen matriculados en cualquier programa pregrado de la universidad y que fuesen consumidores activos de sustancias psicoactivas.

Una vez definidos los invitados, se socializó con ellos el objetivo del estudio y el fin de la información que se recogería. Se realizaron ocho sesiones en profundidad o grupos de enfoque (Hernández-Sampieri et al., 2014, p. 408). Durante todas las sesiones participaron doce estudiantes con el objetivo de conversar a profundidad sobre sus experiencias de consumo de sustancias psicoactivas, en particular todo lo relacionado con el consumo dentro de la universidad, los espacios y actividades asociadas al consumo y los elementos que intervienen en la interacción durante la práctica. Las sesiones fueron grabadas solo en audio, con la intensión de recuperar los aportes y comentarios realizados durante los encuentros; lo anterior con el consentimiento informado de los participantes. Al respecto, es importante aclarar que los nombres que aparecen citados en los fragmentos de las entrevistas son ficticios, a fin de proteger la identidad de los participantes.

La riqueza de la información llevó a la necesidad de profundizar con entrevistas semiestructuradas individuales para comparar las experiencias de consumo y, sobre todo, los aspectos relacionados con las motivaciones e intenciones para el consumo de sustancias psicoactivas, en especial en los espacios de la universidad. Los datos recogidos con los diferentes instrumentos fueron analizados con apoyo del software Atlas.ti (Scientific Software Development GmbH, versión 7) ${ }^{3}$.

\section{Codificación de datos}

A partir de la información recolectada se realizó un proceso de inducción analítica con el objetivo de identificar atributos o propiedades que permitieran hablar de las motivaciones e intenciones para el consumo de sustancias psicoactivas.

Cuando hacemos inducción analítica, desarrollamos y testeamos nuestra teoría caso por caso. Formulamos una explicación del primer caso en cuanto reunimos información al respecto. Aplicamos esa teoría al segundo caso cuando obtenemos información sobre él. Si la teoría lo explica de manera adecuada y, por consiguiente, confirma la teoría, no hay problema; pasamos al tercer caso. Cuando nos topamos con un «caso negativo», uno que nuestra hipótesis explicativa no explica, cambiamos la explicación de lo que intentamos esclarecer, agregándole esos nuevos elementos que nos sugieren los hechos de este caso problemático, o bien cambiamos la definición de lo que vamos a explicar a fin de excluir el caso recalcitrante del universo de cosas a explicar (Becker, 2011, p. 248).

En este sentido se llevó a cabo un proceso de substrucción (Becker, 2011; Ragin, 2007), con el que se trataba de encontrar, para un sistema dado de tipos, el espacio de atributos al que pertenecían los datos y la reducción que se había utilizado implícitamente. El proceso queda ilustrado en la tabla 1.

3 Licencia de la Universidad de Caldas, instalada en el Laboratorio de Estudios Sociales Comparados (Lesc). 


\section{Tabla 1. Proceso de substrucción}

\begin{tabular}{|c|c|c|}
\hline $\begin{array}{c}\text { Guía de acercamiento a la realidad } \\
\text { social }\end{array}$ & Proceso de substrucción & Atributos o propiedades \\
\hline \multirow{6}{*}{$\begin{array}{l}\text { Propósitos o intenciones: se entienden } \\
\text { como "cualquier acto del cual un } \\
\text { agente sepa (crea) qué puede esperar } \\
\text { algo. Cualquier acto que manifieste } \\
\text { una cualidad, un resultado particular } \\
\text { y en el cual éste conocimiento sea } \\
\text { utilizado por el actor con el fin } \\
\text { de producir esta cualidad o este } \\
\text { resultado» (Giddens, 1987, p. 99) }\end{array}$} & $\begin{array}{l}\text { Experimentar sensaciones: hace referencia a las } \\
\text { sensaciones que se buscan con el consumo. } \\
\text { Cuando se consumen sustancias psicoactivas se } \\
\text { potencializan los sentidos y se refiere el mayor } \\
\text { disfrute de actividades cotidianas }\end{array}$ & $\begin{array}{l}\text { - Excitación sexual. } \\
\text { - Sentir llenura. } \\
\text { - Reírse. } \\
\text { - Comer: saborear. } \\
\text { - Tacto: sentir placer; bienestar. } \\
\text { - Escuchar. } \\
\text { - Oler. } \\
\text { - Vista: colores, brillo. }\end{array}$ \\
\hline & $\begin{array}{l}\text { Experimentar emociones: respuestas o } \\
\text { reacciones ante el consumo de psicoactivas. } \\
\text { Estímulos del individuo cuando percibe un } \\
\text { objeto, persona, lugar, suceso o recuerdo } \\
\text { importante. }\end{array}$ & $\begin{array}{l}\text { - Locura. } \\
\text { - Alucinaciones. } \\
\text { - Sobresaltos. } \\
\text { - Delirios. }\end{array}$ \\
\hline & $\begin{array}{l}\text { Cambiar estados de ánimo: para influir en la } \\
\text { manera de percibir el mundo. Olvido de una } \\
\text { situación problemática. }\end{array}$ & $\begin{array}{l}\text { - Estar feliz. } \\
\text { - Desestresarse. } \\
\text { - Relajarse. } \\
\text { - Distraerse. } \\
\text { - Olvidar depresión. } \\
\text { - Olvidar la rabia. } \\
\text { - Olvidar la ira. } \\
\text { - Olvidar los problemas familiares, } \\
\text { laborales, de la universidad, etc. }\end{array}$ \\
\hline & $\begin{array}{l}\text { Relacionarse con otros: el consumo de } \\
\text { psicoactivas como una oportunidad para } \\
\text { interactuar con otros sujetos. }\end{array}$ & $\begin{array}{l}\text { - Construir lazos de amistad. } \\
\text { - Relacionarse. } \\
\text { - Divertirse. } \\
\text { - Pasar bueno. } \\
\text { - Encontrarse con los parceros. } \\
\text { - Compartir. } \\
\text { - Encajar en un grupo. } \\
\text { - Enfiestarme. } \\
\text { - Celebraciones especiales. }\end{array}$ \\
\hline & $\begin{array}{l}\text { Rendir en actividades académicas y cotidianas: el } \\
\text { consumo de psicoactivas como una oportunidad } \\
\text { para hacer las cosas de una mejor manera, } \\
\text { aprovechar el tiempo y ser productivo. }\end{array}$ & $\begin{array}{l}\text { - Rendir al realizar deportes. } \\
\text { - Rendir en el ejercicio intelectual. } \\
\text { - Concentrarse. } \\
\text { - Comprensión de las clases. } \\
\text { - Comprensión de los textos. } \\
\text { - Creatividad. } \\
\text { - Rendir en el trabajo. } \\
\text { - Rendir en el estudio. } \\
\text { - Hacer mejor las cosas. }\end{array}$ \\
\hline & $\begin{array}{l}\text { Intenciones curativas: hace referencia al uso de } \\
\text { las psicoactivas en el orden medicinal. }\end{array}$ & $\begin{array}{l}\text { - Quitar el sueño. } \\
\text { - Provocar el sueño. } \\
\text { - Calmar el dolor. } \\
\text { - Calmar problemas estomacales. }\end{array}$ \\
\hline $\begin{array}{l}\text { Razones: "pueden definirse como } \\
\text { principios fundamentados de la acción } \\
\text { con lo que los agentes se mantienen } \\
\text { en contacto como elemento de rutina } \\
\text { del control reflexivo de sus conductas» } \\
\text { (Giddens, } 1987, p .85)\end{array}$ & $\begin{array}{l}\text { Contexto universitario: hace referencia al } \\
\text { contexto donde ocurren las prácticas y los } \\
\text { elementos que las habilitan o constriñen. }\end{array}$ & $\begin{array}{l}\text { - Consumo de sustancias psicoactivas. } \\
\text { - Percepción sobre el consumo de } \\
\text { psicoactivas. } \\
\text { - Normativa frente al consumo de } \\
\text { sustancias psicoactivas. } \\
\text { - Aplicación de la norma frente al } \\
\text { consumo. } \\
\text { - Espacios de consumo. } \\
\text { - Acceso a las sustancias psicoactivas. }\end{array}$ \\
\hline $\begin{array}{l}\text { Motivos: «hacen referencia a deseos } \\
\text { de los que un actor puede o no ser } \\
\text { consciente, o sólo puede volver } \\
\text { consciente después de haber realizado } \\
\text { el acto al que un motivo particular se } \\
\text { refiere» (Giddens, 1987, p. 117) }\end{array}$ & $\begin{array}{l}\text { Hacen referencia a los elementos que se articulan } \\
\text { con la intención de la acción. } \\
\text { Los motivos responden a deseos, necesidades, } \\
\text { intereses y elementos externos. }\end{array}$ & $\begin{array}{l}\text { - Experimentar sensaciones. } \\
\text { - Experimentar emociones. } \\
\text { - Cambiar estados de ánimo. } \\
\text { - Relacionarse con otros. } \\
\text { - Rendir en las actividades académicas y } \\
\text { cotidianas. } \\
\text { - Curar padecimientos. }\end{array}$ \\
\hline
\end{tabular}


Este ejercicio demandó estrategias en la manera como se ordenaron los argumentos y explicaciones, ya que cada vez que se construyó un concepto, se realizó un proceso de inducción analítica que debió demostrarse. Lo anterior permitió dar cuenta de los aspectos que se incluyen en los procesos de estructuración de las prácticas de consumo de sustancias psicoactivas entre los universitarios entrevistados. A continuación, se presentan los resultados de la indagación.

\section{Resultados: una experiencia de estructuración de las prácticas de consumo de sustancias psicoactivas entre universitarios}

\section{Ilustración 2. Estructuración de las prácticas de consumo de sustancias psicoactivas en universitarios}

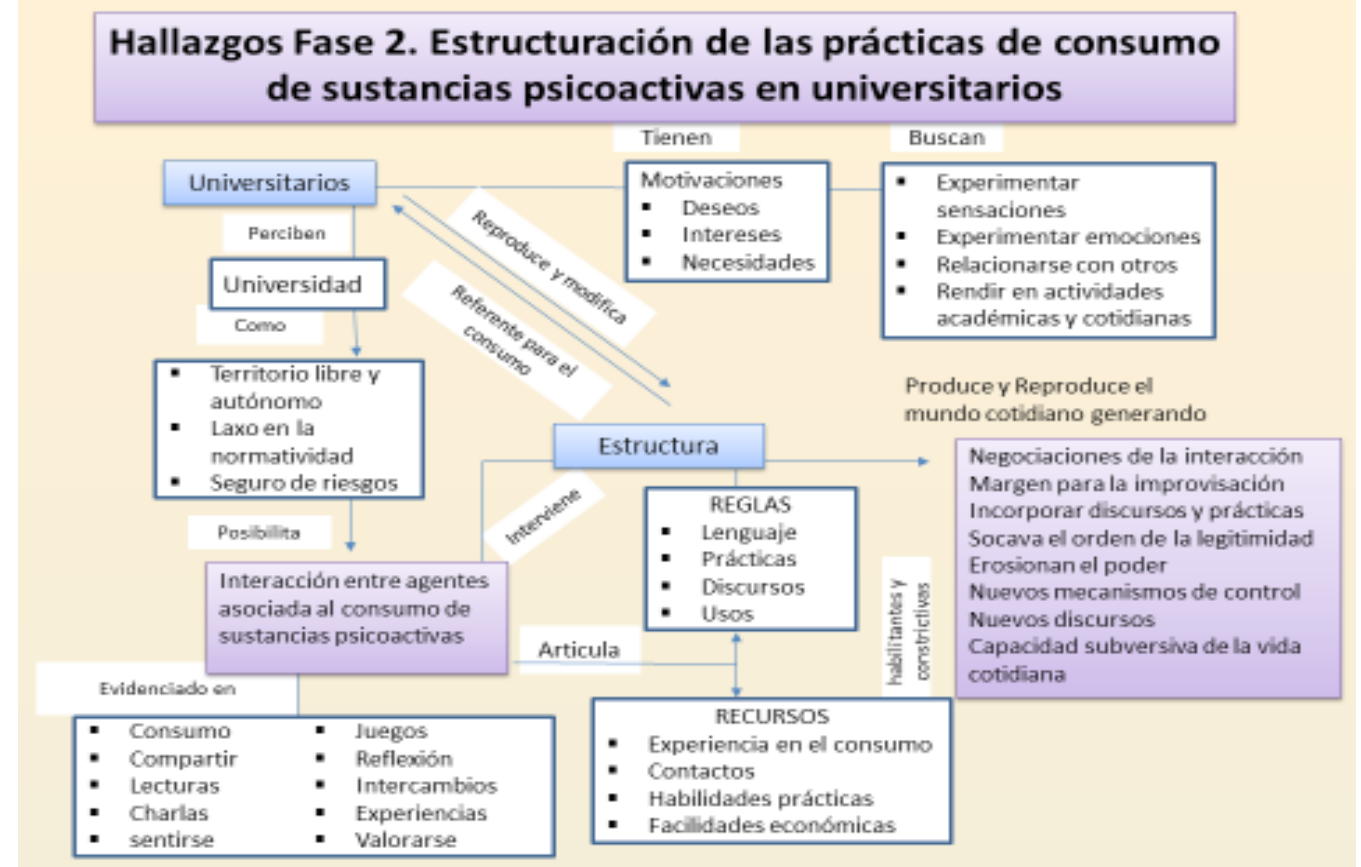

Nota. Elaborada a partir de Giddens (2003).

\section{Elementos individuales en relación con el consumo de sustancias psicoactivas}

Teniendo en cuenta que, desde la teoría de la estructuración, el elemento individual ocupa un lugar importante en la explicación del consumo de sustancias psicoactivas, en el estudio culminado se hizo evidente que la historia personal y social de los universitarios respecto a este tipo de sustancias interviene en el consumo dentro del contexto universitario. Cuando los diferentes escenarios de socialización son flexibles y permisivos frente al tema, y si los inicios del consumo de estas sustancias se presentan a edades tempranas, existe una alta probabilidad de que el consumo continúe y se incremente en el contexto de la universidad. Lo anterior, sin dejar de reconocer la importancia que tienen otros elementos como las circunstancias y el medio social en que se produce la primera experiencia con sustancia psicoactivas, los recursos económicos disponibles, el acceso a las sustancias y su precio, las creencias y mitos existentes frente a los efectos de una sustancia concreta, entre otros aspectos.

En este orden de ideas, el estudio confirmó que las trayectorias de consumo de sustancias psicoactivas comenzaron entre el rango de edad promedio del fin de la secundaria y el inicio de los estudios universitarios, con un consumo que transitó desde formas experimentales hasta formas ocasionales y sociales. Estas prácticas se relacionan principalmente con el deseo de encajar con el 
grupo de pares (otorgándole particular importancia a los amigos y a la pareja), y con la experiencia de un cambio en el estado de ánimo. Sin embargo, llama la atención que la disminución de las edades en el debut del consumo de sustancias psicoactivas es una tendencia mundial y, en determinados contextos, se aprecia la disposición a romper la clásica escalada en el uso de sustancias psicoactivas ${ }^{4}$, de modo que hay quienes inician directamente con sustancias de prescripción médica, marihuana o incluso con cocaína. Al respecto se constató que los medicamentos, en particular los psicofármacos, tienen un peso importante en el inicio del consumo de drogas en el contexto universitario. Las razones para esta situación se encuentran asociadas con el bajo costo de los medicamentos, la percepción de que no son sustancias ilícitas, la característica de que no dejan olor, se consiguen fácilmente, son fáciles de portar y más difíciles de detectar.

Coincidiendo con los autores revisados, según Cuartas (1998) y Parra (1996) el inicio del consumo de sustancias psicoactivas en la universidad también se asocia con la conquista de la autonomía frente al distanciamiento con otros ámbitos sociales como la familia, la escuela y el trabajo, o la soledad de los jóvenes. Esta autonomía es entendida por los entrevistados como el quiebre de los controles familiares y la oportunidad para los excesos, las actividades y actitudes adultas que encuentran justificación y argumentos en la condición de ser universitarios.

Los resultados de la investigación amplían la importancia de los espacios de ocio para los jóvenes y las relaciones interpersonales con el grupo de pares. En este sentido, los jóvenes participan en una gran cantidad de prácticas (entre ellas el consumo de sustancias psicoactivas) como formas características de relacionarse, vivir y pensar. Se confirma además que, siguiendo el deseo de querer ser aceptados en nuevos grupos, los universitarios que no han tenido contacto con sustancias psicoactivas acceden a las dinámicas grupales y paulatinamente se van involucrando en interacciones que desembocan en el inicio del consumo.

Los testimonios evidencian que la entrada a la universidad y los primeros años de vida universitaria se corresponden con un incremento en el consumo y en la diversificación o tránsito a otras sustancias. La diversificación o policonsumo es un rasgo característico, sobre todo entre los consumidores habituales. En muchos casos existe una sustancia principal que hace habitual el consumo (la marihuana para el caso de los universitarios entrevistados) y unas sustancias secundarias que la complementan o la sustituyen en situaciones especiales o cuando no está disponible (LSD, cocaína, poppers). Lo anterior propicia la continuidad en el consumo, debido al lugar que ocupan estas prácticas en el imaginario de ser universitario y en el vínculo social que sus dinámicas facilitan. En este sentido, el inicio del consumo en la etapa escolar, mucho más experimental y casual, se aprecia entre los universitarios como una oportunidad de ensayo; una experiencia que, con frecuencia, se encuentra presente en las formas de relacionamiento y dinámicas particulares de la vida universitaria. Así, quienes ingresan a la universidad con un acercamiento previo a las sustancias psicoactivas, encuentran en el mundo universitario un espacio permisivo para diversificar la experiencia a otro tipo de sustancias y aumentar la frecuencia del consumo.

Si bien la asociación no aparece en todos los casos, en este estudio existe una articulación entre un mayor consumo de marihuana y la baja percepción de riesgo hacia esta sustancia. Lo anterior coincide con la Comisión Interamericana para el Control del Abuso de Drogas (2013), cuando plantea que, entre los países participantes en el II Estudio Epidemiológico Andino sobre consumo de sustancias psicoactivas en población universitaria, Colombia presenta la mayor tasa de consumo de marihuana en la región Andina y, a la vez, los estudiantes colombianos presentan la menor percepción de riesgo frente al consumo de esta sustancia, amparados en argumentos como la no-dependencia, no-embriaguez, el que se percibe como una medicina natural que no tiene químicos y sus usos terapéuticos.

$4 \quad$ Para comprender el modo en que un sujeto se inicia o se mantiene en el uso de drogas se han generado diferentes modelos; entre los más conocidos encontramos el modelo propuesto por Kandel y colaboradores denominado «hipótesis de la puerta», el cual hace referencia al hecho de que el uso de una droga es de algún modo el paso previo para el uso de otras drogas (Kandel \& Jessor, 2002). Este proceso de escalada en el uso de drogas es frecuente, pero no significa una cadena causal en la que las experiencias precoces con drogas legales sean la causa de un uso posterior de drogas ilegales. 
La marihuana se considera entre los universitarios como un estilo de vida, una situación compartida que se asocia con la percepción de formar parte de algo; en palabras de Becker (1963), una subcultura, un conjunto de nociones y puntos de vista acerca de lo que es el mundo, así como rutinas basadas en esas nociones. En este sentido, los entrevistados consumidores de esta sustancia tienden a racionalizar su postura frente al consumo y elaboran argumentaciones que explican por qué consumir no está mal, en tanto no quieren sentirse estigmatizados o señalados por una práctica que, aunque creciente, sigue siendo ilegal, asunto que es mucho más visible si el consumo es individual. Asimismo, se confirma que las prácticas de consumo de sustancias psicoactivas se identifican con la necesidad de interactuar dentro y fuera de la institución y vincularse con actividades recreativas o lúdicas. A partir de lo anterior, los estudiantes se denominan a sí mismos como consumidores sociales y usadores de marihuana.

Ahora bien, las prácticas de consumo de sustancias psicoactivas también se relacionan con procesos de estigmatización y señalamiento. La estigmatización es confirmada entre los estudiantes, quienes advierten que en ocasiones reciben un trato que los iguala a vagos, delincuentes o viciosos ${ }^{5}$. Estas estigmatizaciones pasan también por la influencia que ejercen las construcciones sobre los papeles de género y están relacionadas con los imaginarios edificados a través de la historia, que determinan una carga simbólica mucho más negativa hacia la mujer consumidora.

Los hallazgos evidencian que no puede considerarse que el consumo esté motivado por la desinformación, en tanto las entrevistas afirman que, en general, los estudiantes universitarios poseen conocimientos adecuados acerca de las sustancias psicoactivas y sus efectos. Contrario a lo que plantea la Comisión Interamericana para el Control del Abuso de Drogas (2013), el consumo no tiene como base, al menos en los universitarios de la muestra, problemas socioeconómicos; más bien se trata de motivaciones que se asocian con el deseo de sentirse bien y la necesidad de mejorar algunos aspectos personales que intervienen en la interacción con otros. Lo anterior se confirma en diferentes estudios (Cuartas, 1998; Henao, 2010; Pérez \& Vásquez, 2000).

Como elemento concluyente puede mencionarse que los jóvenes llegan al mundo universitario con condiciones culturales y socioeconómicas diferentes, sin embargo, la vida universitaria homogeniza las prácticas y suspende las condiciones diferenciadas. Así, el consumo de sustancias psicoactivas es una práctica que se naturaliza en el contexto universitario, haciéndose este suficientemente fuerte como para anular las diferencias, homogenizar las prácticas sociales y estructurar o reestructurar las prácticas de una manera diferente a la esperada. Como consecuencia se genera una subcultura de lo universitario, un conjunto de nociones y puntos de vista acerca de lo que es el mundo, así como rutinas basadas en esas nociones.

\section{Motivaciones y propósitos del consumo}

A partir de la información obtenida de los grupos focales, las entrevistas y los ejercicios realizados con los estudiantes que participaron en este trabajo, se identificaron categorías que permiten hablar de las motivaciones y propósitos que tienen los universitarios con respecto al consumo de psicoactivas, elemento importante dentro de la estructuración de este tipo de prácticas. Lo anterior permitió abrir la discusión acerca de lo que quieren y buscan los chicos al consumir ciertas sustancias, advirtiendo sobre la relación que se da, por un lado, entre el consumo y el deseo de experimentar emociones y sensaciones placenteras; y, por otro lado, la relación entre el consumo y la necesidad de mejorar el rendimiento en actividades académicas y cotidianas.

Dado que los motivos «hacen referencia a deseos de los que un actor puede o no ser consciente, o sólo puede volver consciente después de haber realizado el acto al que un motivo particular se

$5 \quad \mathrm{Al}$ respecto debe establecerse una diferenciación entre el consumo de sustancias psicoactivas entre universitarios y las problemáticas relacionadas con las adicciones, el delito y otras situaciones que en ocasiones convergen en el modo de vida en la calle. Ambos son fenómenos y circunstancias de diferente naturaleza para el orden social, y generan distintas consecuencias para la sociedad. Por lo tanto, resulta poco útil tratar de comprender las prácticas de consumo de los estudiantes universitarios, tomando como referencia un problema como el que se menciona. 
refiere» (Giddens, 1987, p. 117), los hallazgos de esta investigación identifican que fue difícil para los universitarios hablar de sus motivaciones para el consumo de sustancias psicoactivas, en tanto se mezclaban con las argumentaciones de los propósitos de la acción. En este sentido, la categoría motivaciones para el consumo de sustancias psicoactivas fue inferida a partir de las intenciones expresadas por los estudiantes, pero se considera que este elemento deja una puerta abierta a nuevas investigaciones sobre el tema.

Se confirma que algunas de las motivaciones más frecuentes del inicio en el consumo están relacionadas con características propias de las edades en que suele ocurrir el primer consumo: la curiosidad, la búsqueda de nuevas experiencias, las creencias acerca de que estas sustancias les ayudarán a ser más activos, creativos y populares y que probarlas no les causará daño. La mayoría de las motivaciones para explicar el inicio en el consumo están generalmente asociadas con una primicia experimental, intermitente y el uso recreativo de estas sustancias. Lo anterior teniendo en cuenta que el ocio es un tiempo de relevancia para todo ser humano y que en estas edades se transcurre preferiblemente con amigos y parejas en espacios informales, fuera del alcance y control de los adultos no consumidores.

Posteriormente, cuando las prácticas de consumo se hacen recurrentes, las motivaciones se convierten en deseos, necesidades e intereses, dando paso a la pregunta sobre la intencionalidad del consumo (¿para qué consumes?), la cual fue explicada a los estudiantes como el propósito del consumo; esta sería aclarada con la pregunta ¿qué buscas cuando consumes una sustancia psicoactiva? La codificación inicial permitió agrupar algunos aspectos que se muestran en la ilustración 3.

\section{Ilustración 3. Intencionalidades del consumo en universitarios}

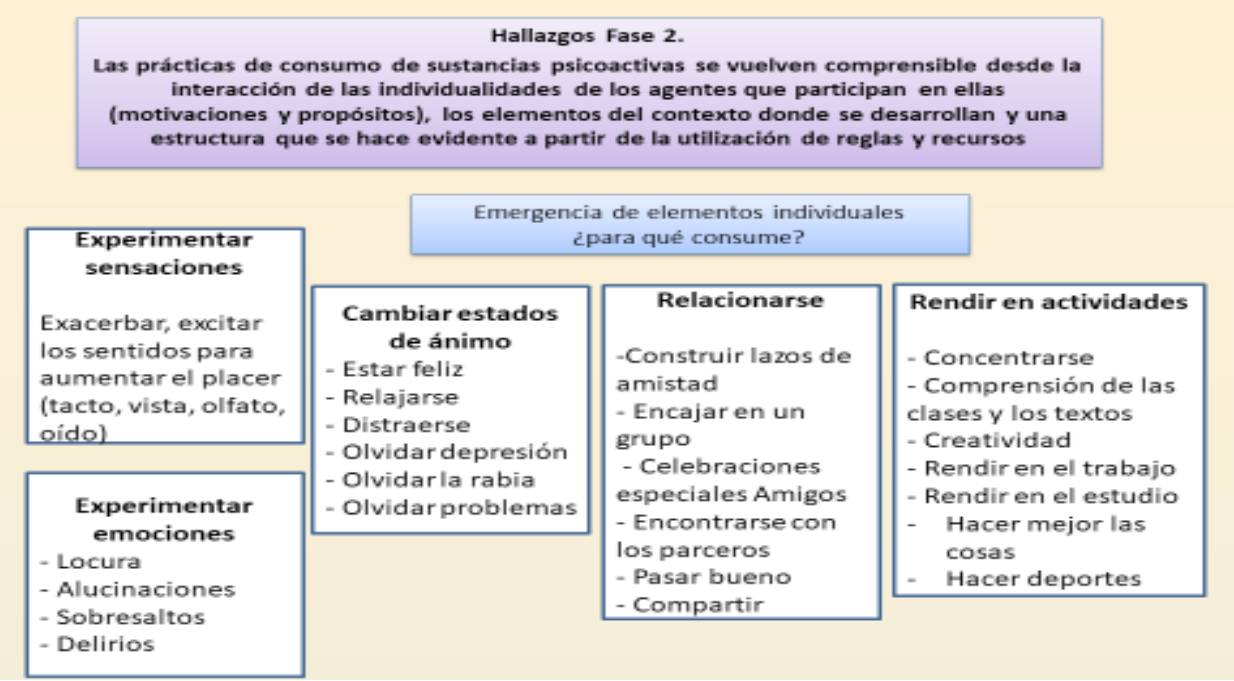

Nota. Elaborada a partir de Giddens (2003).

El análisis de estos elementos permite hablar del interés que tienen los universitarios de integrarse al grupo de pares y la necesidad de autoafirmación dentro de estos. Asimismo, aparece el deseo de facilitar el contacto social, el interés de mostrar más audacia, el deseo de sentirse eufórico, el deseo de desinhibirse para aumentar las habilidades para la conquista y el placer sexual, la necesidad de rendir académicamente, el interés por compartir o percibir experiencias sensoriales o emocionales, entre otras. Aquí, la relación entre universitarios es un aspecto importante, en tanto se constituye en un elemento imprescindible para la vida universitaria. En este sentido, el consumo de sustancias psicoactivas fue descrito como una oportunidad para interactuar y relacionarse con otros sujetos jóvenes ya que permite desinhibirse, ponerse a tono con el ambiente y con los compañeros, divertirse, tener valor para tomar riesgos y quedar bien con el resto del grupo. Asimismo, el consumo de sustancias 
como marihuana se asocia más a la idea de compartir, encontrarse con los parceros, construir lazos de amistad, charlar, reflexionar y pasar un buen momento.

Los jóvenes refieren que, por lo general, consumir marihuana entre amigos es una práctica que conecta a los participantes dado que la dinámica consiste en pasarla bien, relajarse y disfrutar, lo que según los entrevistados los convierte de alguna manera «en parte de una familia». Con las dinámicas asociadas al consumo comienzan a generarse amistades alrededor de las historias, las experiencias que se tejen, las prácticas y todo eso hace parte de ser universitario, hace parte de transitar por la universidad.

\section{Reglas y recursos contenidos en las prácticas asociadas al consumo de sustancias psicoactivas}

En la fundamentación del conocimiento que se va produciendo en el mundo social, aparece una noción de práctica que incluye reglas que no dependen de sujetos ni voluntades, sino que se van estructurando a partir de un juego de estrategias en el espacio-tiempo de la interacción; así como también cualidades que Giddens (2003) denomina recursos, mientras que Bourdieu (2000, pp. 108110) las llama capitales valorados dentro del campo. Así, las reglas y recursos se van reconstituyendo en la interacción social, estableciendo saberes y formas de relacionarse con los otros miembros del grupo.

En el consumo de psicoactivas entre universitarios, algunos de los recursos identificados se relacionan con el conocimiento y la experiencia (conocimiento sobre las sustancias, sus efectos, la experiencia en las combinaciones y sus efectos, la frecuencia de consumo, la dosis adecuada). Lo anterior se constituye en un recurso de autoridad que permite orientar a otros dentro del grupo, al tiempo que genera un valor reconocido en quien lo trae. Otro recurso identificado es tener el contacto con la persona que vende la marihuana, lo cual es un recurso muy valorado dentro del campo en que se mueven los consumidores; otro es la habilidad para armar el porro ${ }^{6}$.

El recurso económico es uno valorado en el campo del consumo, dado que la materia prima para armar los cigarrillos (cueros, cripa) debe comprarse. En la universidad en estudio, por recibir estudiantes en su mayoría de estratos bajo-medio y con pocas posibilidades económicas por sus condiciones mayoritariamente dependientes, tener dinero es un recurso valorado dentro del escenario; sin embargo, con la tendencia en aumento del autocultivo de marihuana, las prácticas de compartir lo que se trae de la casa o regalar un moño aparecen también en aumento. Tener un moño traído de su propia mata es un recurso muy valorado dentro de los miembros del grupo por diversas razones: es gratis, no tiene químicos y genera alrededor del proveedor la imagen de cumplir con uno de los aspectos básicos de la marihuana: compartir con el otro.

Existen recursos en las prácticas de consumo de sustancias psicoactivas, pero también existen reglas que se van reconstituyendo en la interacción entre quienes participan de las prácticas. Estas reglas se orquestan desde la lógica del orden relacional y práctico; en su mayoría son reglas invisibles, nadie las pone, pero todas las conocen y las siguen. Una de las reglas más importantes para el consumo es compartir el porro sin importar quien sea; no se niega porque la lógica es compartir con los amigos, compartir conocimientos, experiencias. Por ello, entre los universitarios fumar acompañado es un elemento importante que cohesiona el grupo y cuyo significado se asocia con estar sintonizado y relajado con los amigos.

Por lo general, cuando se consume en grupos se hace en círculo o en línea dependiendo del espacio. La forma más común para pasar el porro es de uno a otro en el sentido de las manecillas del reloj, permitiendo una integración más fluida. El círculo permite a los participantes estar cara a cara. Resulta particular el hecho de que entre parejas sea común pasarse el humo de boca en boca con un beso.

$6 \quad$ La práctica de armar un porro se adquiere con el tiempo. El porro de marihuana debe tener forma cilíndrica, pero es común que los principiantes tengan dificultades al momento de armarlo en tanto requiere delicadeza y destreza. Después de muchos intentos se arma lo que los jóvenes llaman «empanadas fumables», «pitillo» o «banano». Con el tiempo se va mejorando la técnica, y a los sujetos con la habilidad de armar bien los cigarrillos se les llama «arquitectos». 
Aunque la forma más común de consumir marihuana es armando un cigarrillo, existen otras formas como las pipas, las frutas y los dulces. Las pipas generalmente son de uso individual, aunque pueden usarse en grupo cuando hay poca cripa o se está en espacios inapropiados. La cripa es lo más apropiado para fumar en pipa, por un lado, porque sus efectos se sienten más rápido $\mathrm{y}$, por otro, porque el olor es más suave y fácilmente confundible con el olor del cigarrillo. Fumar en las frutas es similar a la pipa, en tanto se hace una perforación en la fruta, generalmente en bananos, manzanas y piñas, aunque también puede hacerse en zanahorias y papas, allí se abren dos orificios: en uno se coloca el porro y por el otro se fuma. Esta particular forma de fumar se asocia más a los espacios campestres o paseos en grupos.

Las elaboraciones como brownies, chocolates, arroz con leche, tortas, té, pomadas y lápiz labial, entre otras formas, buscan tener los efectos de la marihuana disimulando la indeseada «pizca», esto es, el olor que queda impregnado en el cuerpo después de fumar. Para eliminarlo se utilizan cremas de manos, lociones, gel antibacterial, jabón para manos, incienso, goma de mascar o mentas para el aliento. También existen matanceras, herramienta en forma de pinza que permiten coger el porro y fumarse la pata sin quemarse ni dejar impregnado el olor en los dedos.

Si la sustancia es el popper, las formas de consumir son otras. Por lo general esa sustancia tiene un olor muy fuerte y se inhala. Con frecuencia se aplica en la ropa: unas veces se aplica en el cuello de la camisa como si fuera loción o en la empuñadura de la manga larga, de esa manera el chico puede oler la sustancia constantemente en el cuello o el puño de la ropa. Otras sustancias como los hongos, se mezclan con productos como la leche condensada y bebidas como el agua panela.

\section{Particularidades del contexto universitario en relación con las prácticas asociadas al consumo de sustancias psicoactivas}

Por la importancia del espacio como una forma dinámica de pertenencia, los universitarios entrevistados reconocen e identifican algunas zonas específicas para las prácticas de consumo al interior de la universidad. Algunos de estos espacios se caracterizan por ser abiertos, al aire libre y alejados del tránsito de personas. Este hallazgo es confirmado por el estudio de Red Unir (2009) en el que se evidencian espacios, dentro y fuera de las universidades de Medellín, asociados con las prácticas de consumo de sustancias psicoactivas, que otorgan significados sobre las maneras de habitar, de entender y vivir el paso por la universidad.

Coincidiendo con Henao (2010), para muchos estudiantes la universidad está cargada de emociones que derivan de las experiencias y sentimientos que se generan por la permanencia en estos espacios, representando para ellos su segunda casa. Esta vivencia se relaciona con la sensación de seguridad y protección que les genera la institución. La percepción que tienen los entrevistados sobre la universidad es que se constituye en un espacio propio de quienes lo habitan y se ven a ellos mismos como protagonistas y principales habitantes del escenario, lo cual les otorga, desde sus experiencias, derechos sobre los espacios de la universidad.

Los hallazgos muestran que entre los estudiantes existe el imaginario de que la universidad, por tener carácter público, es un espacio libre más allá de las normas institucionales. Al mismo tiempo, ese carácter de institución universitaria se vive como un territorio al que los dispositivos de la fuerza pública no pueden acceder, constituyéndose en un escenario calmo y tranquilo que los resguarda, evitando que se expongan a los riesgos de la calle, la violencia, redes de microtráfico y abusos policiales, entre otras situaciones negativas.

Se confirma la debilidad de las acciones propuestas (generalmente desde las oficinas de bienestar universitario) para mitigar las prácticas de consumo en las universidades, lo que se refleja también en posiciones institucionales que no son claras frente a la situación de consumo de la población universitaria. Lo anterior se relaciona con la permisibilidad en los controles institucionales y las normativas vigentes frente al tema. Si bien las normativas prohíben el consumo de sustancias psicoactivas como un elemento generador de problemas de convivencia dentro de la institución, el 
tema no es abordado con regularidad o sistematicidad sino hasta que en algún espacio del campus se hace evidente y recurrente el consumo. Estas situaciones generan que, en la medida en que se cierran espacios para evitar el consumo en ellos, los grupos de estudiantes inmersos en estas prácticas se desplazan hacia nuevos escenarios. Estos nuevos espacios se van redefiniendo y resignificando de manera permanente, desde sus experiencias personales y prácticas colectivas.

Esta nueva conquista del espacio se va dando en la medida en que unos actores y prácticas permanecen cada vez más en el territorio y otros por indiferencia, malestar o exclusión evitan hacer presencia en él. En este sentido, la música, los juegos, la apariencia de los concurrentes, la forma de vestir, el color del pelo, el peinado, el tono de la voz, el olor en el espacio, los gestos, el lenguaje, entre otros, son aspectos que van territorializando estos espacios y caracterizando a las personas que los frecuentan. Al respecto, un elemento importante en la estructuración de las prácticas sociales es la coherencia de la posición individual con la grupal, lo que define la inclusión o no en los grupos. En este orden de ideas, la coherencia que se menciona es un aspecto muy importante entre los universitarios; de ahí que las personas se sientan, directa o indirectamente, presionadas a asumir una postura que les permita mantener la armonía consigo mismos y con los demás. Es precisamente esta una de las maneras en la que se estructuran las prácticas entre universitarios: al interior de los grupos se excluyen a aquellos que no comparten intereses similares, y lo contrario a la exclusión es la dinámica de inclusión. Los hallazgos evidencian que los grupos de universitarios se encuentran en una doble dinámica: por un lado, existe una coerción interna, es decir, internamente están en proceso de autoidentificarse y constantemente se están construyendo en la aceptación de otros como ellos, por lo que resulta muy valorado reunirse con los mismos que hacen lo que yo hago; y, por otro lado, excluyen a aquellos cuyos intereses son diferentes y no encajan en el grupo.

Aunque los autores muestran que en las dinámicas y actividades dentro de los grupos de universitarios prevalece el consumo, las conclusiones de la investigación evidencian que existen otras muchas actividades que realizan al momento de reunirse, en las que las sustancias psicoactivas no son el elemento central. En este sentido, se destaca el hecho de que aparecen dos tipos de estudiantes: el que consume y forma parte de las prácticas de consumo y el estudiante que no consume, pero que también participa de las prácticas asociadas a este tipo de sustancias. Lo anterior sugiere pensar que el consumo de sustancias psicoactivas, en especial el consumo de marihuana, se comporta como un dispositivo socializador que permite a los estudiantes vincularse entre sí, compartir experiencias y generar lazos de solidaridad, complicidad y amistad.

\section{El consumo de sustancias psicoactivas: ¿una práctica problemática?}

Algunos abordajes epidemiológicos ubican el consumo de sustancias psicoactivas como una práctica problemática o asociada a comportamientos dependientes o delincuenciales. Como consecuencia, las instituciones universitarias y la política pública en general plantean acciones prohibicionistas hacia el consumo de psicoactivas dentro de las instituciones universitarias. Sin embargo, esta postura implica sanciones que llevan a la estigmatización, a la exclusión del sujeto de determinados espacios y a su desplazamiento a lugares clandestinos.

Los hallazgos de esta investigación evidencian que quienes asumen que la universidad es un espacio público, además de constituirse en un lugar plural, defienden el consumo de psicoactivas en ella e incluso reclaman el territorio como un lugar libre y de libertades individuales, demandando la necesidad de tomar distancia con las redes de microtráfico y promoviendo las prácticas de autocultivo de sustancias como marihuana.

Así mismo, el consumo de psicoactivas entre universitarios, en especial la marihuana, se constituye en un elemento dinamizador alrededor del cual los jóvenes se relacionan para realizar varias actividades a partir del establecimiento de prácticas recurrentes, como un hecho individual o colectivo, que representa intereses comunes. Así, el consumo de sustancias psicoactivas entre 
universitarios puede entenderse como un facilitador del vínculo social y como un mediador que estimula la interacción entre grupos de pares.

Ahora bien, las reflexiones que los chicos realizan sobres sus propios escenarios dentro de la universidad permiten pensar en una variable de poder, como la capacidad de agencia Giddens (2003), así como explicar el consumo como una práctica transgresora y como un ejercicio de reapropiación del escenario. La tesis de la apropiación del espacio universitario, que se configura entre los procesos de producción social del territorio y el consumo de marihuana, se concreta en las estrategias de poder y resistencia que construyen los jóvenes para la defensa de territorios articulados al consumo de sustancias psicoactivas. Dichas estrategias de poder y resistencia se caracterizan por la creatividad, la creación de redes de intercambio, la invención de formas de encubrir los insumos y la variedad de actividades que se dinamizan en estos escenarios. De este modo, el poder no es solamente algo dado, derivado de la estructura en el sentido estructuralista (es decir, algo que no puede ser modificado y que subordina a las personas), sino que, como las prácticas, es producido en las relaciones sociales.

Si bien es cierto que estos grupos de consumidores respetan algunas medidas establecidas por la institución universitaria, se consideran a sí mismos distintos y buscan hacerlo explícito mediante el discurso, las argumentaciones, la apropiación de los espacios y el consumo a la vista de todos, entre otros elementos diferenciadores. En la actualidad -y en unas universidades más que en otras- el tema de la prohibición de sustancias psicoactivas se reduce a lo que está establecido en el reglamento estudiantil; pero no se debaten en el marco de la academia aspectos tan importantes como los usos, las motivaciones, la nocividad, la convivencia, las zonas de consumo y, sobre todo, el asunto de la libertad y el libre desarrollo de la personalidad. Este trabajo confirma que los modelos orientados hacia el control y la prohibición no solucionan los conflictos generados por el consumo de sustancias psicoactivas; por tanto, desde lo complejo del fenómeno, es necesario plantear otros abordajes que lleven a comprender, desde los jóvenes y su necesidad de adaptarse al contexto universitario, las razones para el consumo de psicoactivas dentro del campus.

Estos elementos plantean interrogantes a los estamentos directivos de las instituciones universitarias sobre la responsabilidad en el proceso de formación integral de sus estudiantes y evidencian la importancia de analizar los diferentes factores culturales, políticos y económicos que envuelven, tanto a las sustancias, como a los sujetos que las consumen. En este sentido, en el estudio de la Red Unir (2009) estudiantes y profesores de las universidades públicas de Antioquia y Nacional de Colombia señalan la necesidad de plantear el tema de los consumos de drogas como un asunto de libertades donde los consumidores sean escuchados y se consideren sus construcciones, sentidos y argumentos. Estos son insumos valiosos para tener en cuenta al momento de generar las políticas de intervención de la universidad, como un hecho consecuente con la formación y el respeto por la diferencia.

Por otro lado, en los estudios nacionales o regionales en Colombia aparece la tendencia a homogeneizar el problema de las drogas como si fuera un mismo fenómeno universal, atemporal y ahistórico, cuando se trata de un fenómeno plural con variadas manifestaciones según el momento histórico, la cultura, el modelo económico y la situación particular de cada país. La realidad es que los diversos significados que le asignan los sujetos a las sustancias psicoactivas obedecen precisamente a lo temporal e histórico. Esta mirada aparece en estudios financiados, generalmente por los Estados, donde la tendencia es percibir las drogas como una sustancia prohibida y su uso como una conducta desviada. En algunos casos justificada por una línea policiaca y, en otros, por una línea terapéutica. Henao (2010) plantea que frente a todos estos elementos se avista un mediano conocimiento, por parte de las instituciones, acerca de las múltiples dimensiones que se asocian con el consumo de sustancias psicoactivas en población universitaria; ello se refleja en el desenfoque y poca pertinencia de las acciones y programas, que resultan desarticulados con los procesos de formación y capacitación de la comunidad universitaria en general. 


\section{Conclusiones sobre los procesos de estructuración de las prácticas asociadas al consumo de sustancias psicoactivas en universitarios}

La investigación permite concluir que las prácticas de consumo de sustancias psicoactivas en universitarios se estructuran a partir de la interrelación que se establece entre:

a) la historia personal de los estudiantes frente al consumo de sustancias psicoactivas;

b) las características del contexto donde se dan las prácticas (la universidad) y la postura adoptada por la institución frente a este tipo de prácticas;

c) las motivaciones y propósitos de los universitarios para participar del consumo de sustancias psicoactivas;

d) la interacción entre los estudiantes que participan de este tipo de prácticas.

En este sentido, la interrelación de los elementos contextuales e individuales que se orquestan en las prácticas sociales producen un proceso constante de reproducción de normas y valores, tanto grupales como sociales, que se articulan al consumo de este tipo de sustancias. Así, lo individual contiene la capacidad práctica que tienen los sujetos de realizar una acción a partir de sus motivaciones y propósitos, y los elementos estructurales representan un marco de referencia que se configura en razones que orientan a los jóvenes para el consumo. La interacción que se genera entre los agentes durante la práctica de consumo de sustancias psicoactivas articula reglas y recursos que se van reconfigurando en nuevas formas de conocimiento, establecen saberes y crean nuevas expresiones y formas de relacionarse con otros miembros del grupo.

\section{La entrada a la universidad}

Si bien la universidad, desde su condición dinamizadora de grupos sociales con diferentes culturas, etnias y costumbres en algunas ocasiones se convierte en un foco de inicio al uso de sustancias psicoactivas, el inicio en el consumo aparece con frecuencia antes de llegar a este nivel educativo. Por otra parte, la diversificación o policonsumo es un rasgo característico, sobre todo entre los consumidores habituales.

Hemos identificado a la universidad como un escenario que, por su laxitud y permisibilidad frente al consumo de sustancias psicoactivas, admite por omisión el consumo de sustancias psicoactiva, en particular el consumo de marihuana. Asimismo, se confirma la debilidad de las acciones institucionales frente a la situación de consumo de la población universitaria. Lo anterior se relaciona con la permisibilidad en los controles institucionales y las normativas vigentes frente al tema.

\section{La marihuana}

El consumo de psicoactivas en grupo (en particular la marihuana) tiene un gran contenido simbólico entre quienes las comparten, estructurando vínculos sociales, lazos de confianza y redes que generan nuevas formas de expresarse y relacionarse con otros. La marihuana se percibe entre los universitarios como un estilo de vida, una situación compartida que se asocia con la percepción de formar parte de algo; esto es, una subcultura, un conjunto de nociones y puntos de vista acerca de lo que es el mundo, así como rutinas basadas en esas nociones.

Los entrevistados consumidores de esta sustancia tienden a racionalizar su postura frente al consumo y elaboran argumentaciones que explican por qué consumir no está mal, en tanto no quieren sentirse estigmatizados o señalados por una práctica que, aunque creciente, sigue siendo ilegal.

\section{Las prácticas de consumo}

Un elemento importante en la estructuración de las prácticas es la coherencia de la posición individual con la grupal, lo que define la inclusión o no en los grupos. Al interior de los grupos se 
excluyen a aquellos que no comparten intereses similares y, lo contrario a la exclusión, es la dinámica de inclusión.

Las prácticas de consumo se relacionan con procesos de estigmatización y señalamiento. Estas estigmatizaciones pasan también por la influencia que ejercen las construcciones sobre los papeles de género y están relacionadas con los imaginarios edificados a través de la historia que determinan una carga simbólica mucho más negativa hacia la mujer consumidora.

\section{Las motivaciones}

La mayoría de las motivaciones para explicar el inicio en el consumo están generalmente asociadas con una primicia experimental, intermitente y el uso recreativo de estas sustancias. Posteriormente, las motivaciones se convierten en deseos, necesidades e intereses, entre ellos:

- la necesidad de integrarse al grupo de pares,

- la necesidad de autoafirmación dentro del grupo,

- el deseo de facilitar el contacto social,

- el interés de mostrar más audacia,

- el deseo de sentirse eufórico,

- el deseo de desinhibirse para cumplir con la necesidad de aumentar las habilidades para la conquista,

- el interés por compartir o percibir experiencias sensoriales o emocionales.

Lo anterior advierte que los estudiantes con prácticas recurrentes de consumo buscan intencionalmente experimentar las sensaciones y efectos de estas sustancias, lo que nos ubica en una práctica intencionada y con propósitos claros.

\section{Una práctica trasgresora}

Las prácticas de consumo de sustancias psicoactivas dentro de la universidad advierten sobre la capacidad de agencia de los jóvenes en tanto es posible comprender el consumo de sustancias psicoactivas como una práctica transgresora y como un ejercicio de reapropiación del escenario. También como los jóvenes construyen estrategias de poder y resistencia para la defensa de territorios articulados al consumo; dichas estrategias se caracterizan por la creatividad, creación de redes de intercambio y la invención de formas de encubrir los insumos y la variedad de actividades que se dinamizan en dichos espacios.

Dado que el poder no es solamente algo dado derivado de la estructura, sino que puede ser modificado, en las prácticas descritas el poder es producido en las relaciones sociales, y buscan hacerlo explícito mediante el discurso, las argumentaciones, la apropiación de los espacios, el consumo a la vista de todos, entre otros elementos diferenciadores.

Finalmente, en la producción y reproducción de ese mundo cotidiano se generan negociaciones de la interacción, un margen para la improvisación más allá de las reglas y recursos presentes en la interacción, se incorporan discursos y prácticas, se socava el orden de la legitimidad, se erosiona el poder y se crean nuevos mecanismos de control y nuevos discursos, lo que Rossana Reguillo (2000) llama la capacidad subversiva de la vida cotidiana.

\section{Sugerencias}

Se sugiere la revisión de la normativa institucional frente al consumo de sustancias psicoactivas, en especial frente a la marihuana, con una mirada incluyente que no vulnere el derecho de los universitarios a participar de los territorios de la universidad y su derecho al reconocimiento de las libertades individuales. También es importante debatir en el marco de la academia aspectos tan importantes como los usos, las motivaciones, la nocividad, la convivencia, las zonas de consumo. 
Estos, entre otros elementos, plantean interrogantes a los estamentos directivos de las instituciones sobre la responsabilidad en el proceso de formación integral de sus estudiantes y evidencia la importancia de analizar los diferentes factores culturales, políticos y económicos que envuelven tanto a las sustancias psicoactivas como a los sujetos que las consumen.

Es necesario generar espacios de discusión frente a la temática del uso social de las drogas, como una práctica que se ha ido normalizando en la vida universitaria y en la que confluyen sujetos de diferentes culturas, etnias y clases sociales, provenientes de diferentes partes del país a fin de desestigmatizar al universitario consumidor, en especial de marihuana, y escuchar las diferentes voces.

\section{Lista de referencias}

Becker, H. (1963). Outsiders: hacia una sociología de la desviación. Buenos Aires: Siglo XXI. Becker, H. (2011). Trucos del oficio. Buenos Aires: Siglo XXI.

Bourdieu, P. (2000). La distinción: criterio y bases sociales del gusto. Madrid: Taurus.

Castellanos-Obregón, J. M., \& Espinosa-Herrera, G. (2013). Revisión de las tendencias de investigación sobre consumo de sustancias ilegales por los jóvenes. Virajes, 15(2), 15-55.

Comisión Interamericana para el Control del Abuso de Drogas. (2013). II Estudio epidemiológico andino sobre consumo de drogas en población universitaria. Lima: Autor.

Creswell, J., \& Plano, V. (2011). Designing and conducting mixed methods research. London: Sage.

Cuartas, R. (1998). Apuntes para una reflexión polémica acerca de las sustancias psicoactivas. Cultura y Droga, (1), 1-18.

Espinosa-Herrera, G., Castellanos-Obregón, J. M., \& Osorio-García, D. (2016). Condición juvenil y drogas en universitarios: el caso de una universidad regional. Revista Latinoamericana de Ciencias Sociales, Niñez y Juventud, 14(2), 1451-1468. doi:10.11600/1692715x14238060615

Fernández-Bustos, P. (2010). Estudio de los factores de riesgo y protección del consumo. (Tesis doctoral). Universidad de Alcalá, Alcalá, España.

Franey, C. (1998). Theories of drug and alcohol misuse and intervention, Introduction. London: Imperial College of Science, Technology and Medicine.

Fundación Universitaria del Área Andina. (2011). Consumo de sustancias psicoactivas en estudiantes universitarios. Pereira: Fundación Universitaria del Área Andina.

García-Moreno, M. D. (2002). Consumo de drogas en adolescentes: diseño y desarrollo de un programa de prevención escolar. (Tesis). Universidad Complutense de Madrid, Madrid, España.

Giddens, A. (1987). Las nuevas reglas del método sociológico. Buenos Aires: Amorrortu.

Giddens, A. (2003). La constitución de la sociedad. Buenos Aires: Amorrortu.

Henao, S. (2010). Representaciones sociales del consumo de «drogas»y de las intervenciones respectivas en un contexto local: la Universidad de Antioquia en Medellín, Colombia. (Tesis de doctorado). Universidad de Granada, Granada, España.

Hernández-Sampieri, R., Fernández-Collado, C., \& Baptista-Lucio, M. (2014). Metodología de la investigación. México, D. F.: McGraw-Hill.

Instituto de Adicciones de Madrid. (2012). Estudio sobre consumo de drogas en jóvenes y adolescentes. Madrid: Instituto de Adicciones de Madrid.

Kandel, D., \& Jessor, R. (2002). The Gateway hypothesis revisited. Stages and pathways of drug involvement: Examining the Gateway hypothesis. Nueva York: Cambridge University Press.

López, M. J., Santín, C., Torrico, E., \& Rodríguez, J. M. (2003). Consumo de sustancias psicoactivas en una muestra de jóvenes. Psicología y Salud, 13(1), 5-17.

Oficina de las Naciones Unidas contra la Droga y el Delito. (2013). Informe mundial sobre las drogas 2013. Recuperado de:

https://www.unodc.org/doc/wdr2013/World_Drug_Report_2013_Spanish.pdf 
Oficina de las Naciones Unidas contra la Droga y el Delito. (2016). Informe mundial sobre las drogas 2016. Recuperado de:

https://www.unodc.org/doc/wdr2016/WDR_2016_ExSum_spanish.pdf

Parra, R. (1996). La Universidad. En R. Parra (Ed.), Escuela y modernidad en Colombia (pp. 253257). Bogotá, D. C.: Tercer Mundo.

Patiño, J. (2012). Patrones de consumo de cocaína en los jóvenes. (Tesis doctoral). Universidad de Girona, Girona, España.

Pérez, E., \& Vásquez, C. (2000). Función del consumo de sustancias psicoactivas en la estructuración psíquica del adolescente. (Trabajo de grado). Universidad del Norte. Barrranquilla, Colombia.

Ragin, C. (2007). La construcción de la investigación social: introducción a los métodos y su diversidad. Bogotá, D. C.: Siglo del Hombre.

Red Unir. (2009). Del viaje en U: la vivencia universitaria y el consumo de sustancias psicoactivas. Medellín: Fondo Editorial Funlam.

Reguillo, R. (2000). Emergencia de culturas juveniles: estrategias del desencanto. Buenos Aires: Norma. 